\title{
Thickening of tailings using hydrocyclones operating under vacuum conditions
}

\author{
G.J. van Ryssen Cyclone Projects and Consulting, South Africa \\ P.L. Steenkamp AngloGold Ashanti Ltd, Senegal
}

\section{Abstract}

Many tailings storage facilities (TSFs) on the African continent are constructed by utilising the proven cyclone deposition method. The plant tailings is separated to deliver a coarse sandy underflow to the wall zone of the TSF and the overflow is discharged into the basin. The average percentage of dry gold tailings solids that is available for the wall zone is generally between 20 and 35\%. The product is stable and high rates of rise (RoR) can be achieved. The TSF impoundment wall is normally constructed to one vertical and three horizontal (approximately $18^{\circ}$ ).

A vacuum cyclone system has been developed for a mineral sands operation located close to the sea shore on the South African west coast, to flatten the side slopes to one vertical and five horizontal. The reason for the flat slopes is for rehabilitation of the TSF in this very arid part of the country, which is distressed by high velocity northwesterly and southeasterly winds. The tailings density of the TSF averages $1.17 \mathrm{t} / \mathrm{m}^{3}$ and the conventional approach to cycloning is not feasible. With the vacuum system an average underflow of $1.9 \mathrm{t} / \mathrm{m}^{3}$ is achieved with the overflow density averaging $1.01 \mathrm{t} / \mathrm{m}^{3}$. The solids recovery achieved for wall flattening is approximately $88.5 \%$.

A gold mine in West Africa is utilising this technology in placing approximately 40-50\% of the solids pumped to their TSF on the outer wall in the downstream fashion, delaying the need for a new greenfields facility. The existing TSF will have adequate capacity to accommodate the slimes from the plant for Life of Mine (LoM), thus minimising the risk of overloading the facility.

This paper describes the process of utilising hydrocyclones operated under vacuum conditions to:

- flatten wall slopes for rehabilitation

- increase the capacity of a TSF by placing solids downstream of an existing wall

- buttress a TSF wall, increasing the overall stability.

\section{Introduction}

The use of cycloning techniques is a well-known practise in constructing perimeter embankments for TSFs wall zones in the African continent during the life of the facility. This technology, which involves separating coarse particles from a tailings stream, is however, impractical for tailings streams containing less than $30 \%$ solids. A mineral sands operation in South Africa has in its closure plan the need to flatten the side slopes of its TSFs. One of the options considered was to doze the $30 \mathrm{~m}$ high walls to the required slope of 1:5. The saving in costs by not dozing was probably the biggest incentive when contemplating cycloning as an alternative option, provided that the tailings stream could be separated successfully in delivering adequate underflow volumes before mine closure. The required cyclone underflow recovery rate was calculated to be $70 \%$. Cyclone testing done some time before proved that a recovery rate of $74 \%$ was achievable with a feed density which averaged $1.31 \mathrm{t} / \mathrm{m}^{3}$. This was much higher than the density actually received during commissioning. 
Two cyclone cluster units were designed for the full stream of $172 \mathrm{t} / \mathrm{hr}$ solids, generated from the plant. During commissioning of the units it was discovered that the feed density to the TSF dramatically changed to less than $1.20 \mathrm{t} / \mathrm{m}^{3}$ versus the higher density received during the testing work.

\section{The vacuum cyclone cluster unit}

In order to allow for portability and flexibility, two cluster units were designed and manufactured namely; one comprising $10 \times 250$ diameter polyurethane lined hydrocyclones and the second unit comprising five similar cyclones. Changes were made to the cyclones inner configuration, spigot discharge and overflow in order to create a vacuum in each cyclone. Each unit was fitted with an underflow discharge collection launder from which the coarse tailings could be directed to the point of deposition. Collective overflow pipes were fitted to each cluster to discharge the water and fine tailings into the basin of the TSF.

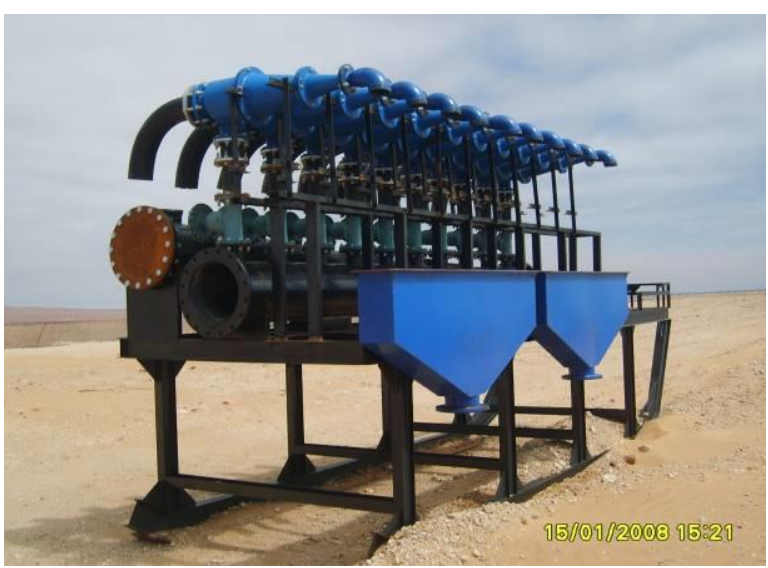

(a)

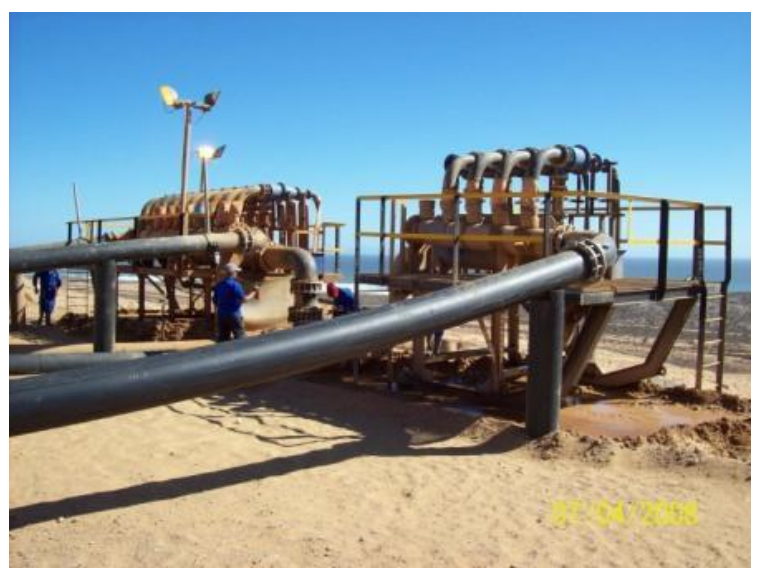

(b)

Figure 1 (a) cluster before changing to vacuum system; (b) operational vacuum system

\section{Slope flattening}

A launder system was constructed using old conveyor belts sourced from the mining scrap yard. The intention was to commence with the placing of thickened underflow at the toe of the dam, progressing to the crest of the dam. Once the first section had been completed, the cluster units would be moved to a position adjacent to the previous position, and the deposition process repeated. During the planning and designing phase, the option was kept open to use a dozer from time to time to create paddock walls. It was found that due to the ease with which the cluster units were operated, the underflow density was controlled to deliver material suitable for placing and developing the slope without the need to doze.

The density and recovery graphs in Figures 2 and 3 give a visual image of the vacuum cyclone cluster performance.

Higher than calculated underflow recovery rates were achieved throughout and more solids were placed downstream of the walls at the required slope.

Due to the success achieved, the mine has recently ordered four additional units which are designed to treat the total residue stream. 


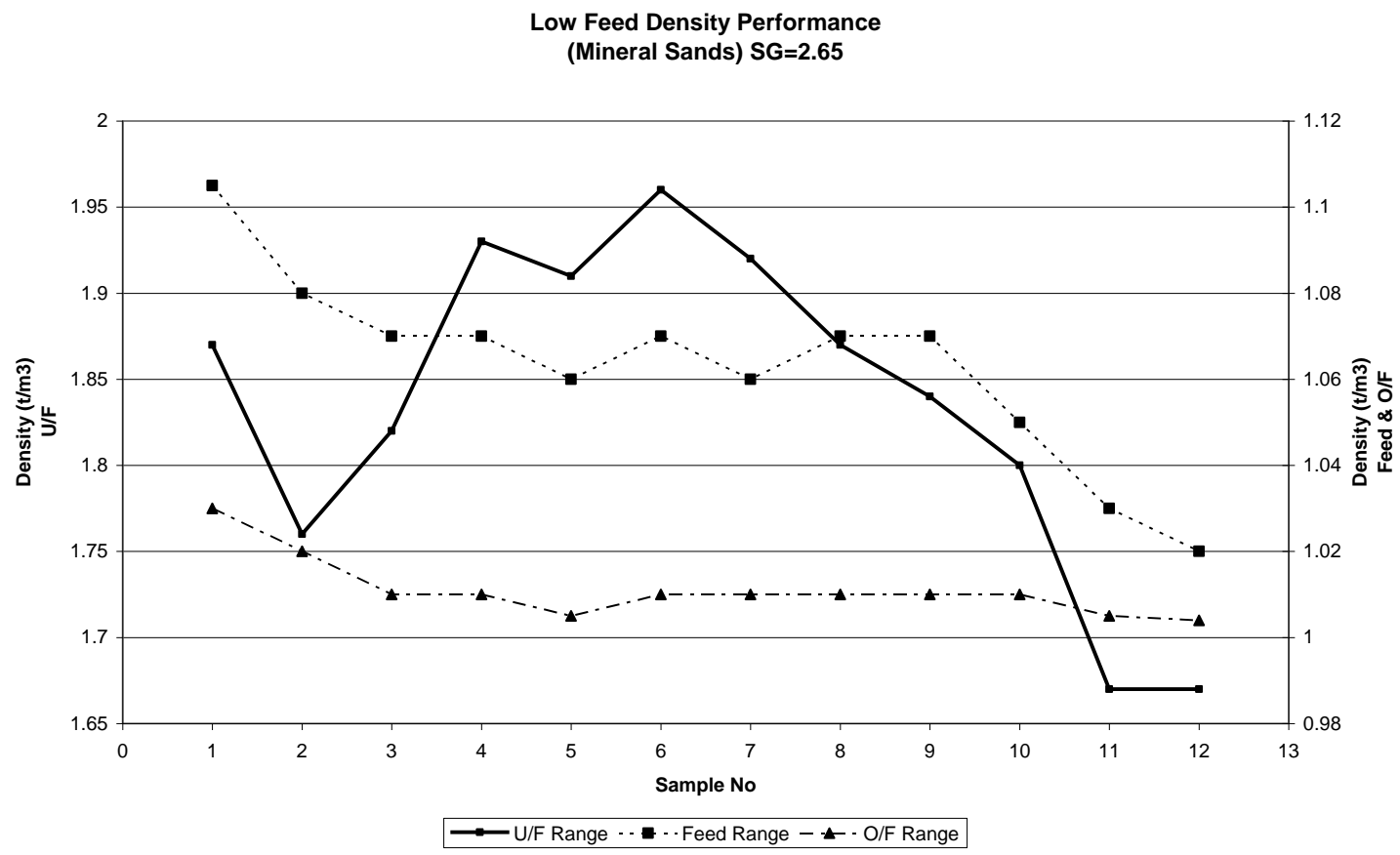

Figure 2 Density chart for low density vacuum cyclone feed

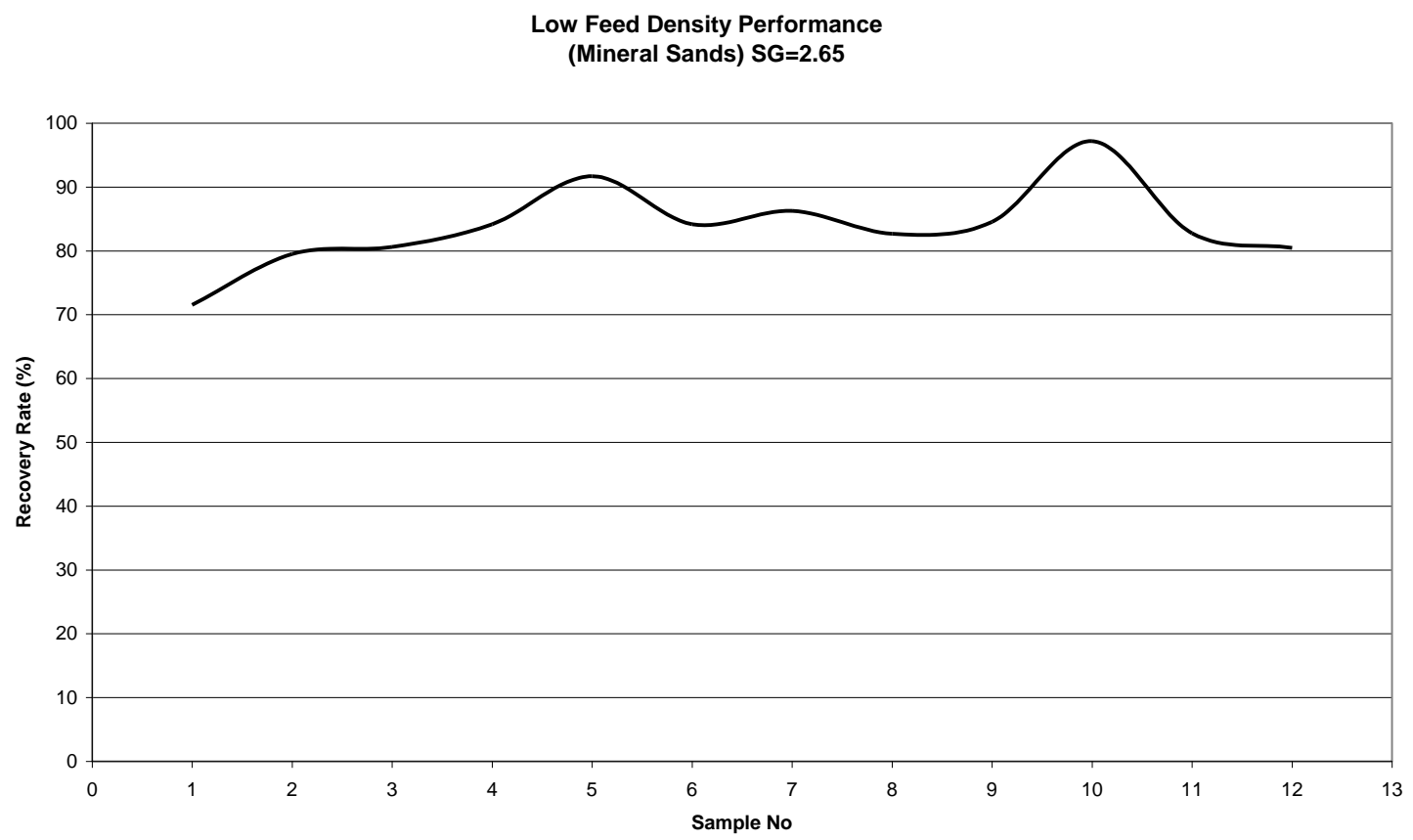

Figure 3 Recovery performances for low density vacuum cycloning 


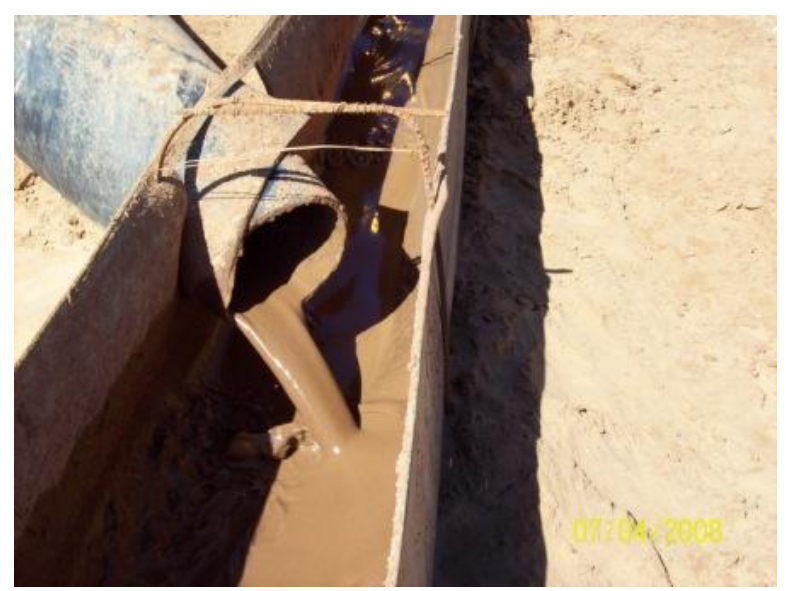

(a)

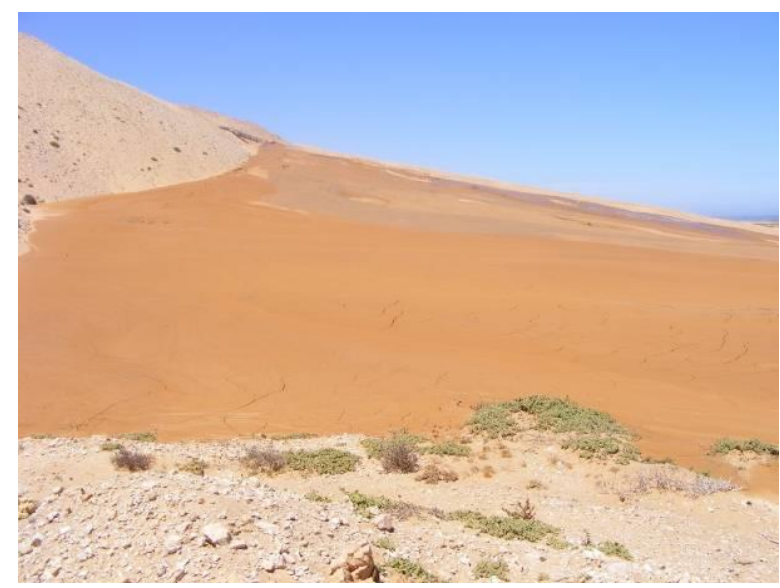

(b)

Figure 4 (a) Launder systems for underflow transportation; (b) slope flattening in progress

\section{$4 \quad$ The use of vacuum cycloning for increasing TSF capacity}

The principle of vacuum cycloning was extended to the gold mining industry. The need arose at a gold mine in Western Africa to increase the TSF capacity by placing more material to the downstream side of the main valley wall. Apart from increasing the capacity, the stability of the wall would be increased due to a massive buttress that would be created by this action. Due to the sensitivity of the area and the client's no pollution policy, the vacuum system had to be designed to allow the maximum percentage solids to the underflow and therefore the highest possible liquid to the overflow. Testing work on the system commenced during August 2009 and was completed during April 2010. The underflow properties can easily be changed to deposit a buttress at any slope required. The percentage solids in the underflow vary between 75 and $80 \%$. No bleed water is visible and the minimum subsoil drainage is required.

The testing work proved that a large percentage of the tailings being pumped to the TSF could safely be placed on the downstream side of the main wall, thereby increasing the capacity and extending the lifetime of the existing TSF. The vacuum system would also be utilised for straightening and flattening the existing walls for mine closure.

Many TSFs around the world show signs of stability stress. Large volumes of rock are often placed adjacent to the specific area at high cost to the mine. By utilising this vacuum cycloning principle, large volumes of solids can be placed downstream of the walls, at a low cost, to create a buttress on slopes which are also suitable for rehabilitation.

The density and recovery graphs below give a visual image of the vacuum cyclone testing work performance. 
Medium Feed Density Performance (Gold Slimes) SG=2.73

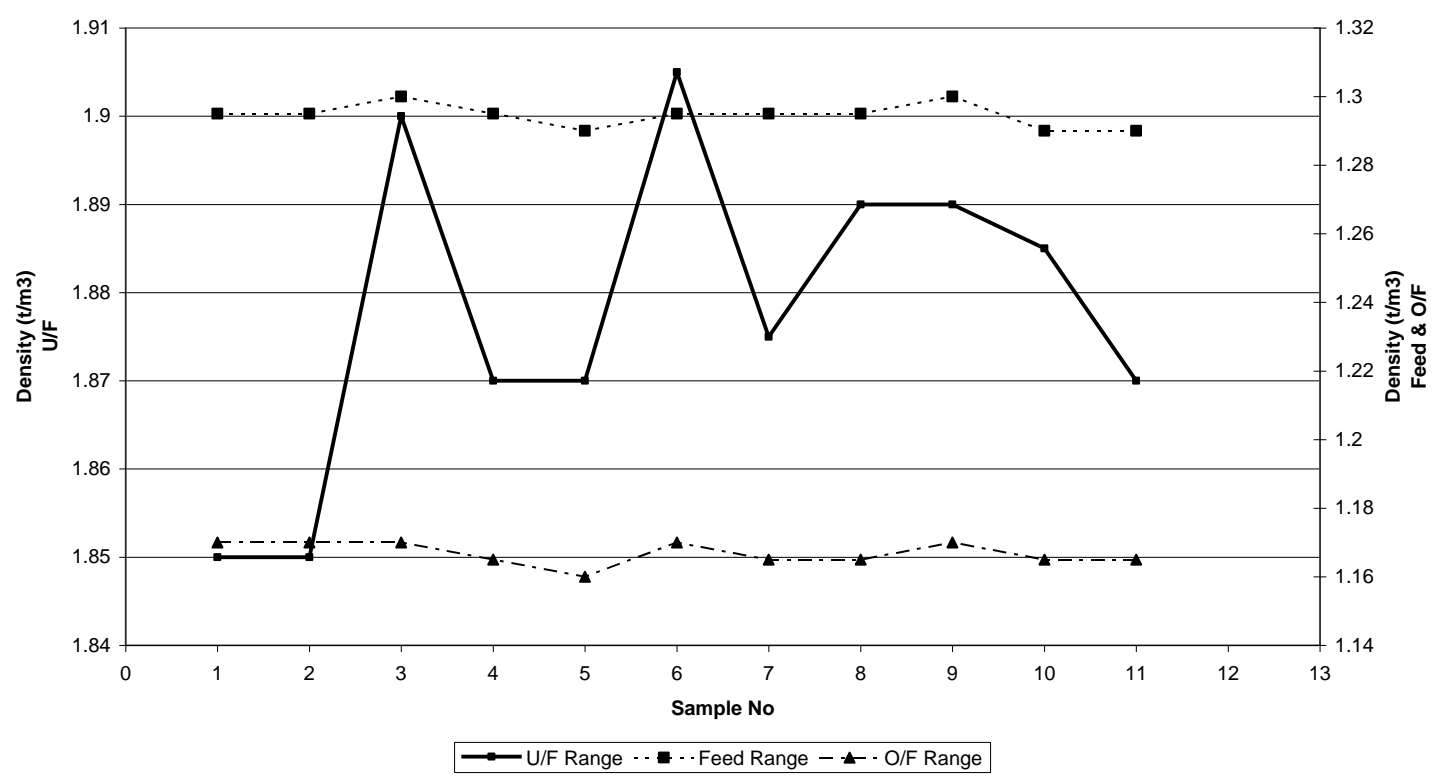

Figure 5 Density chart for medium density vacuum cyclone feed

Medium Feed Density Performance (Gold Slimes) SG=2.73

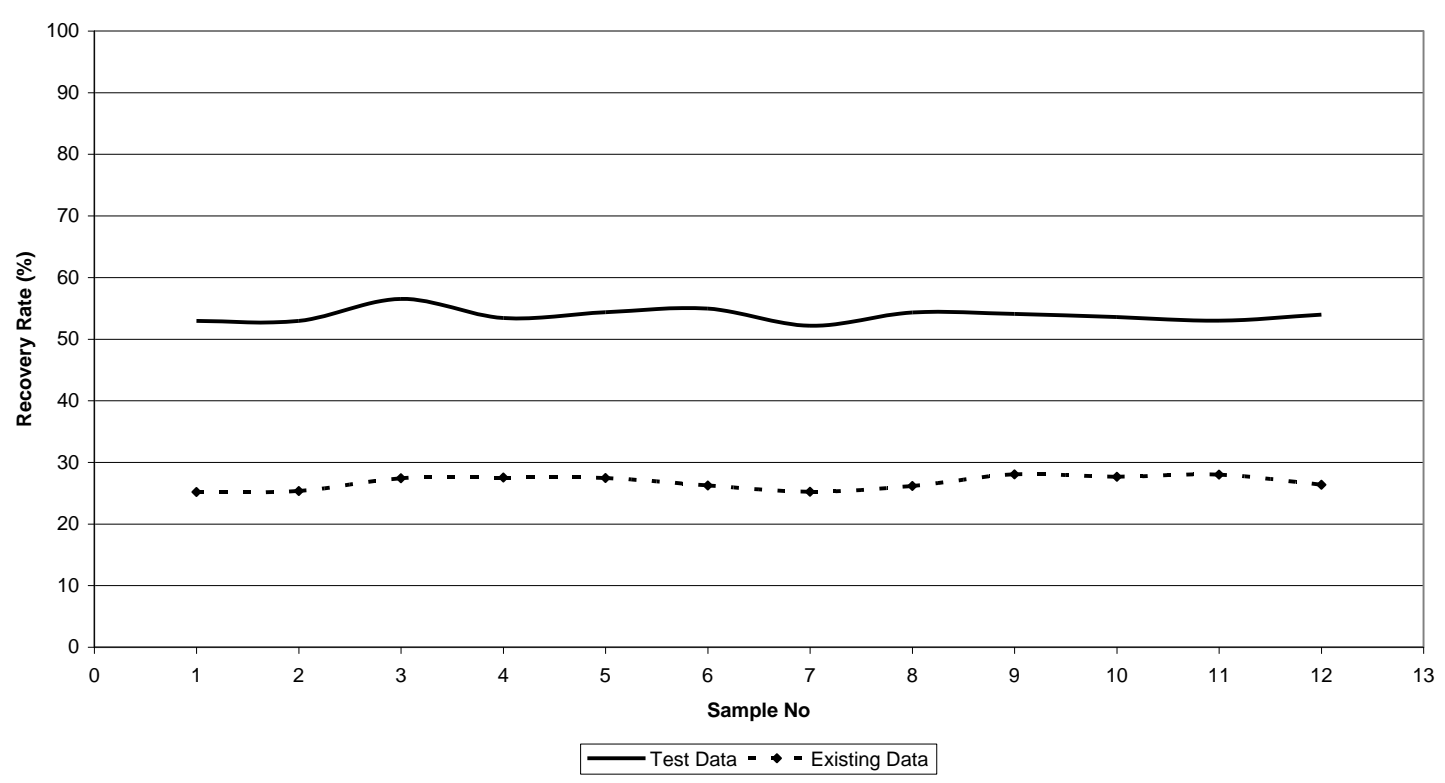

Figure 6 Recovery performances for medium density vacuum cycloning 


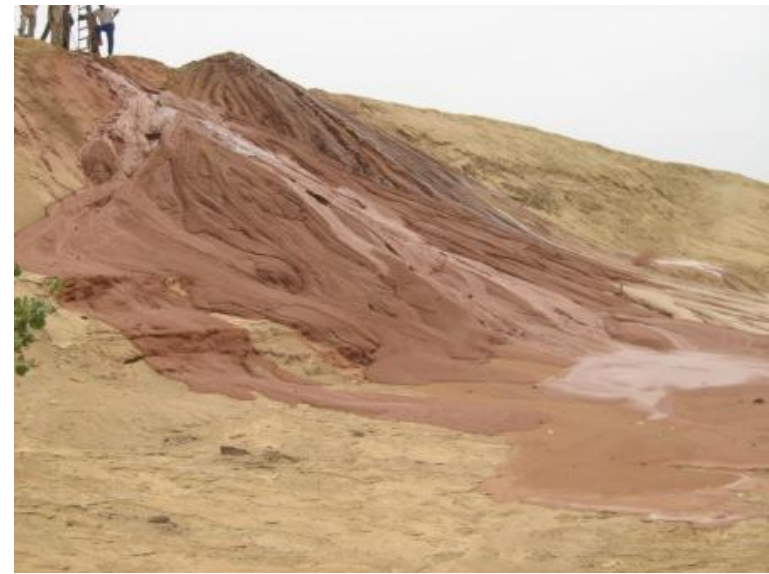

(a)

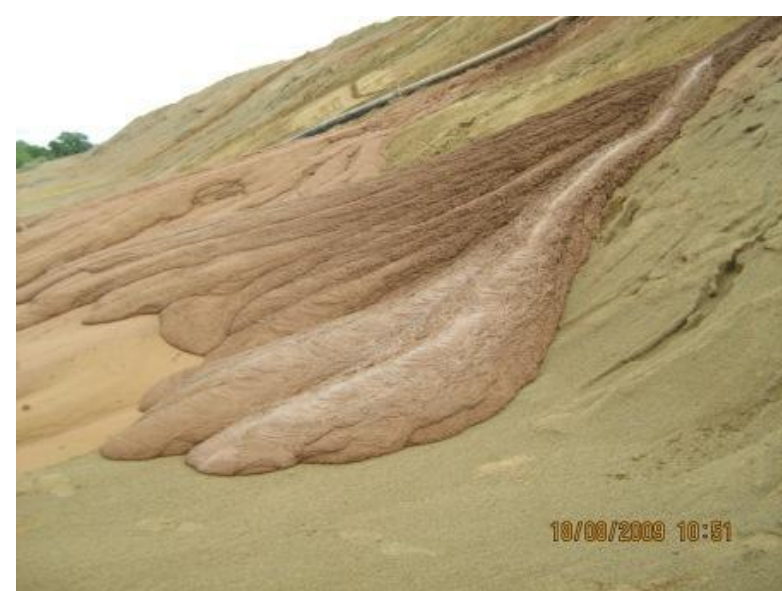

(b)

Figure 7 (a) Different slope configurations; (b) high solid content - no bleed water

\section{$5 \quad$ The use of vacuum cycloning for increasing TSF stability and capacity}

The TSF of another mine in Western Africa is located in the remains of a crater. Due to the geotechnical properties of the tailings, a laterite starter wall was constructed in order to contain the tailings and raised by another $6 \mathrm{~m}$ using the downstream method. Conventional upstream cycloning was introduced to replace a third downstream laterite wall raise. The cycloning is a major success, but the risk to stability remains an issue. The placing of a massive buttress downstream of the laterite wall by utilising vacuum cycloning, has commenced.

Apart from increased stability, another benefit would be the increase in capacity. The existing TSF has adequate capacity for another two years. Greenfield sites have been identified for new TSF construction. After thorough investigation and volumetric analysis during April 2010, it was determined that adequate capacity could be created for an additional eight years on the existing TSF by increasing the footprint, utilising the vacuum cycloning system. An additional $130 \mathrm{Mm}^{3}$ could be added by downstreaming along the entire perimeter of the 567 ha facility, increasing the capacity from the designed elevation of 414 mamsl to 435 mamsl.

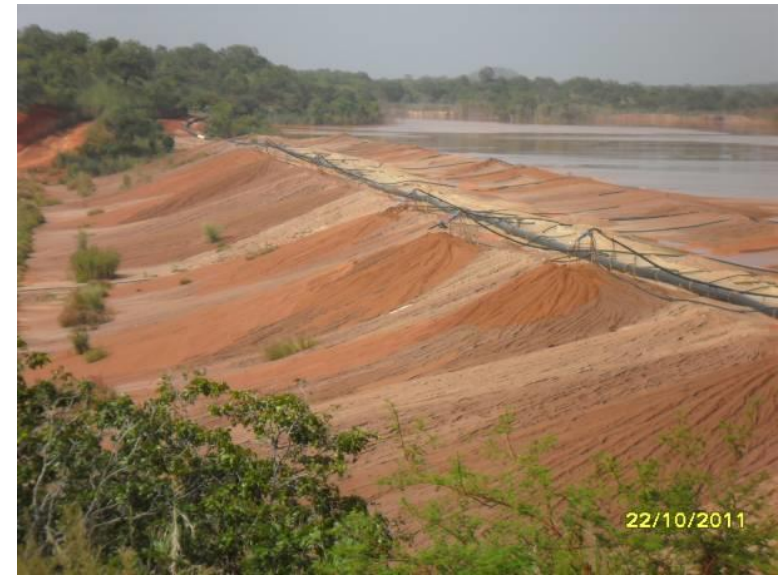

(a)

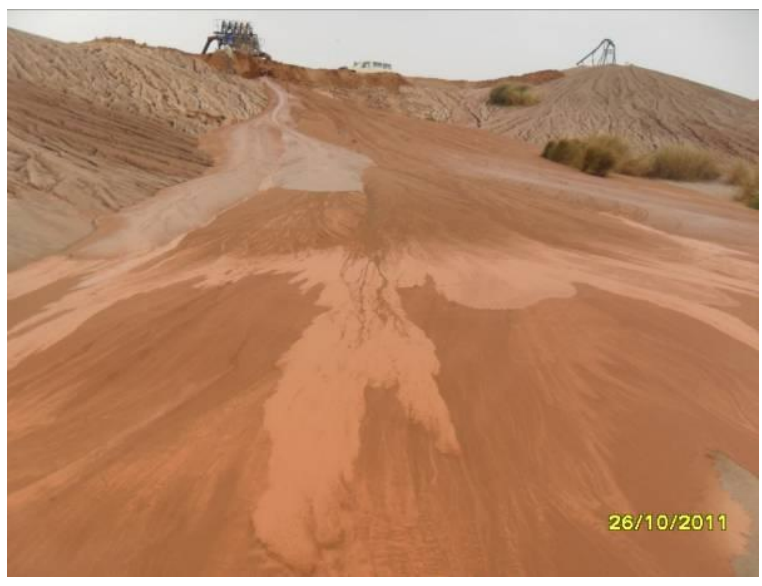

(b)

Figure 8 (a) cycloned wall; (b) vacuum cycloning during commissioning of a cluster 


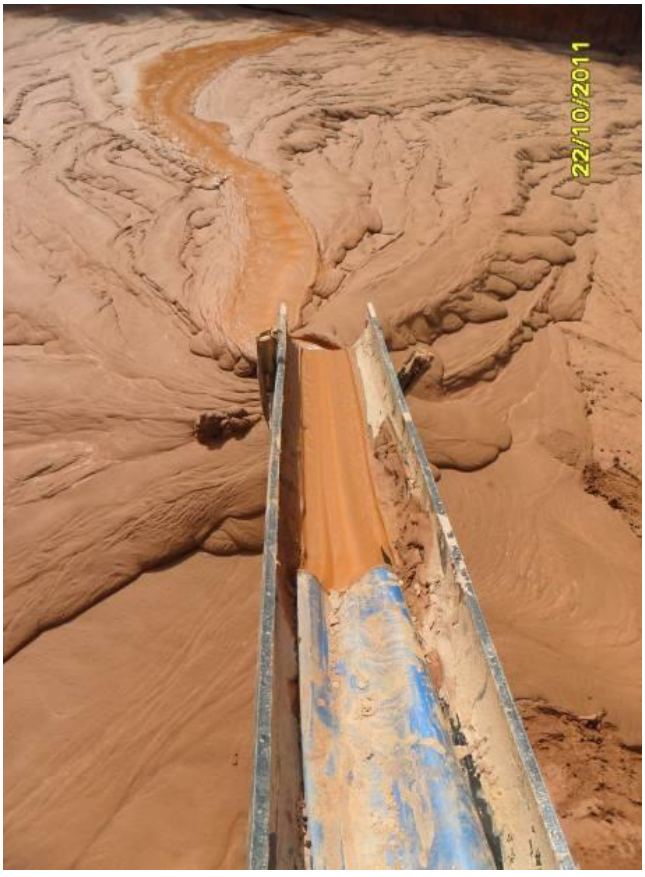

(a)

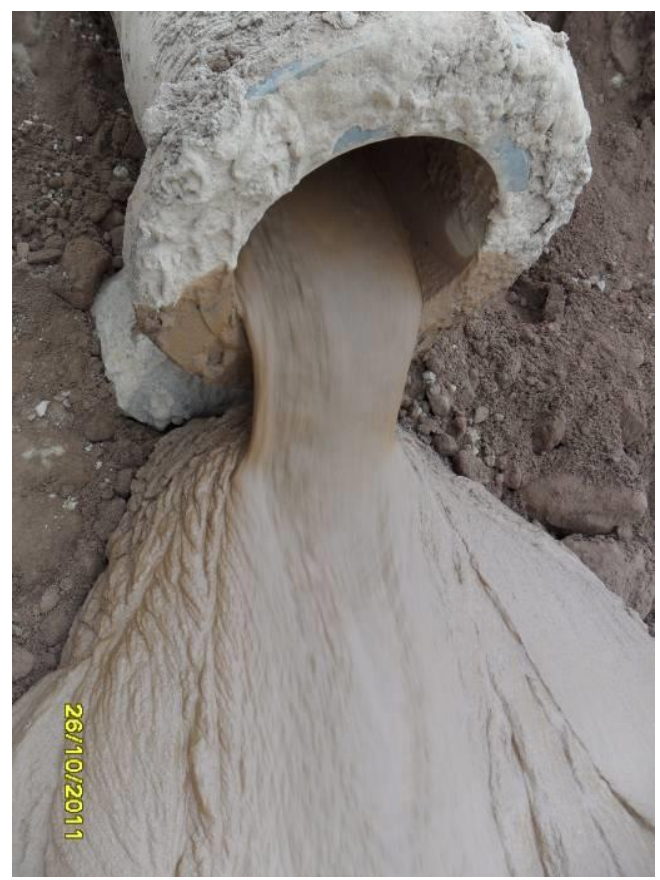

(b)

Figure 9 (a) vacuum cycloning during testing; (b) vacuum cyclone product

\section{The change over from an existing cyclone TSF to vacuum cycloning}

Changing from an existing cyclone TSF to vacuum cycloning can be done without interference of the process plant or TSF operations. The change over process entails manufacturing the cluster structure/s and cluster piping. Existing cyclones utilised on the TSF are re installed on the cluster structure and the final inner adjustments are completed for vacuum cycloning. Underdrainage construction between the finer base and coarser cyclone material can be done without any interruption of dam operations.

\section{$7 \quad$ Conclusions}

The method and significance of the vacuum cyclone technology have been presented. The use of vacuum cycloning on new or existing TSFs has economic advantages which can be summarised as follows:

- Increased stability on an unsound zone of the wall at a fraction of the cost of placing a rock buttress.

- Side slope flattening of steep walls during the operational phase for rehabilitation and closure.

- Increased capacity of existing TSFs. 
